\title{
Fejér type integral inequalities related with geometrically-arithmetically convex functions with applications
}

\author{
S. S. Dragomir, M. A. Latif, and E. Momoniat
}

\begin{abstract}
A new identity involving a geometrically symmetric function and a differentiable function is established. Some new Fejér type integral inequalities, connected with the left part of Hermite-Hadamard type inequalities for geometrically-arithmetically convex functions, are presented by using the Hölder integral inequality and the notion of geometrically-arithmetically convexity. Applications of our results to special means of positive real numbers are given.
\end{abstract}

\section{Introduction}

The classical convexity is defined as follows.

A function $f: I \rightarrow \mathbb{R}, \emptyset \neq I \subseteq \mathbb{R}$, is said to be convex on a convex set $I$ if the inequality

$$
f(t x+(1-t) y) \leq t f(x)+(1-t) f(y)
$$

holds for all $x, y \in I$ and $t \in[0,1]$.

A vast literature has been reported on inequalities concerning classical convexity during the past three decades, but the most celebrated inequalities in mathematical analysis for convex functions are the Hermite-Hadamard inequalities

$$
f\left(\frac{a+b}{2}\right) \leq \frac{1}{b-a} \int_{a}^{b} f(x) d x \leq \frac{f(a)+f(b)}{2},
$$

where $f: I \rightarrow \mathbb{R}$ is a convex mapping and $a, b \in I$ with $a<b$. The inequalities in (1.1) hold in upturned direction if $f$ is a concave function.

Received April 3, 2018.

2010 Mathematics Subject Classification. Primary 26D15; Secondary 26A51; 26E60; $41 \mathrm{~A} 55$.

Key words and phrases. Hermite-Hadamard's inequality; Fejér's inequality; convex function; GA-convex function; Hölder's inequality; power mean inequality.

http://dx.doi.org/10.12697/ACUTM.2019.23.05 
In the past few years the theory of convex sets and convex functions have been a subject of extensive research and these notions have been generalized and extended in diverse directions. As a result many new proofs, noteworthy extensions, generalizations, refinements, new Hermite-Hadamard type inequalities and numerous applications of the inequalities (1.1) have arisen in the literature of mathematical analysis and in various other branches of pure and applied mathematics.

We begin with the following generalization of the usual or classical convexity which is called the geometrical-arithmetical convexity or GA-convexity.

Definition 1 (see $[7,8])$. Suppose that $I \subseteq \mathbb{R}_{+}=(0, \infty)$. A function $f: I \rightarrow \mathbb{R}$ is said to be GA-convex on $I$ if

$$
f\left(x^{\lambda} y^{1-\lambda}\right) \leq \lambda f(x)+(1-\lambda) f(y)
$$

holds for all $x, y \in I$ and $\lambda \in[0,1]$, where $x^{\lambda} y^{1-\lambda}$ and $\lambda f(x)+(1-\lambda) f(y)$ are, respectively, the weighted geometric mean of two positive numbers $x$ and $y$, and the weighted arithmetic mean of $f(x)$ and $f(y)$.

The notion of GA-convexity is further generalized as GA-s-convexity in the second sense as follows.

Definition 2 (see [4]). Let $s \in(0,1]$. A function $f: I \rightarrow \mathbb{R}$ is said to be GA-s-convex on $I \subseteq \mathbb{R}_{+}$if

$$
f\left(x^{\lambda} y^{1-\lambda}\right) \leq \lambda^{s} f(x)+(1-\lambda)^{s} f(y)
$$

holds for all $x, y \in I, \lambda \in[0,1]$.

Most recently, İşcan [4] proved the following result for GA-s-convex functions.

Theorem 1 (see [4]). Suppose that $f: I \rightarrow \mathbb{R}$ is GA-s-convex in the second sense, and $a, b \in I \subseteq \mathbb{R}_{+}$with $a<b$. If $f \in L[a, b]$, then

$$
2^{s-1} f(\sqrt{a b}) \leq \frac{1}{\ln b-\ln a} \int_{a}^{b} \frac{f(x)}{x} d x \leq \frac{f(a)+f(b)}{s+1} .
$$

If $f$ in Theorem 1 is GA-convex function, then we get the inequalities

$$
f(\sqrt{a b}) \leq \frac{1}{\ln b-\ln a} \int_{a}^{b} \frac{f(x)}{x} d x \leq \frac{f(a)+f(b)}{2} .
$$

In [6], the authors introduced the notion of geometrically symmetric functions as follows.

Definition 3 (see $[6]$ ). A function $g:[a, b] \rightarrow \mathbb{R},[a, b] \subseteq \mathbb{R}_{+}$, is said to be geometrically symmetric with respect to $\sqrt{a b}$ if

$$
g\left(\frac{a b}{x}\right)=g(x), \quad x \in[a, b] .
$$


The authors in [6] also proved the following Fejér type integral inequalities, which provide a weighted generalization of (1.3).

Theorem 2 (see [6]). Let $f: I \rightarrow \mathbb{R}$ be a GA-convex function and let $a, b \in I \subseteq \mathbb{R}_{+}$with $a<b$. If $g:[a, b] \rightarrow[0, \infty)$ is a continuous, positive, and geometrically symmetric to $\sqrt{a b}$ mapping, then

$$
f(\sqrt{a b}) \int_{a}^{b} \frac{g(x)}{x} d x \leq \int_{a}^{b} \frac{f(x) g(x)}{x} d x \leq \frac{f(a)+f(b)}{2} \int_{a}^{b} \frac{g(x)}{x} d x .
$$

Several new integral inequalities were also established in [6] for the right part of the inequality (1.4), which not only provide weighted generalization of the results from [4] connected with the right part of (1.3), but also give refinements of those results for particular choice of the geometrically symmetric weight functions.

For more results on Hermite-Hadamard type inequalities concerning GAconvex functions, $s$-GA-convex functions and their applications we refer the reader to $[1]-[6],[9],[10]$, and references therein.

In Section 2, we will prove a weighted integral identity for the left part of the inequality (1.4) involving a differentiable mapping and a geometrically symmetric function. We will use this identity, the geometrical-arithmetical convexity and some auxiliary results to obtain some new Fejér type integral inequalities related with the left part of (1.4). The results of Section 2 provide a weighted version of the results given in [4] for the left part of the inequality (1.3) and also refine those results for particular choice of the geometrically symmetric function involved. Some applications of our results to special means of positive real numbers are given in Section 3.

\section{Fejér type integral inequalities for geometrically-arithmetically convex functions}

Throughout this paper we will use the notations

$$
U(t):=a^{(1-t) / 2} b^{(1+t) / 2}, \quad V(t):=a^{(1+t) / 2} b^{(1-t) / 2} .
$$

The beta function and the integral from of the hypergeometric function are defined as follows to be used in the sequel of the paper:

$$
B(\alpha, \beta):=\int_{0}^{1} t^{\alpha-1}(1-t)^{\beta-1} d t, \alpha>0, \beta>0
$$

and, for $|z|<1, \gamma>\beta>0$,

$$
{ }_{2} F_{1}(\alpha, \beta ; \gamma ; z):=\frac{1}{B(\beta, \gamma-\beta)} \int_{0}^{1} t^{\beta-1}(1-t)^{\gamma-\beta-1}(1-z t)^{-\alpha} d t .
$$

In addition, for numbers $a>0$ and $b>0$ with $a \neq b$ let

$$
A(a, b):=\frac{a+b}{2}, L(a, b):=\frac{b-a}{\ln b-\ln a}, G(a, b):=\sqrt{a b}, H(a, b):=\frac{2 a b}{a+b},
$$


and

$$
L_{p}(a, b):= \begin{cases}{\left[\frac{b^{p+1}-a^{p+1}}{(p+1)(b-a)}\right]^{\frac{1}{p}},} & p \neq-1,0, \\ L(a, b), & p=-1, \\ \frac{1}{e}\left(\frac{b^{b}}{a^{a}}\right)^{\frac{1}{b-a}}, & p=0,\end{cases}
$$

be the arithmetic mean, logarithmic mean, geometric mean, harmonic mean, and generalized logarithmic mean of order $p \in \mathbb{R}$, respectively.

Now we prove a weighted integral identity which plays a key role in establishing our main results.

Lemma 1. Let $f: I \rightarrow \mathbb{R}, I \subseteq \mathbb{R}_{+}$, be a differentiable function on $I^{\circ}$ and let $a, b \in I^{\circ}$ with $a<b$. Suppose that $g:[a, b] \rightarrow[0, \infty)$ is a continuous, positive, and geometrically symmetric to $\sqrt{a b}$ mapping. If $f^{\prime} \in L([a, b])$, then the following equality holds:

$$
\begin{aligned}
& \mathcal{F}(f, g ; a, b):=f(\sqrt{a b}) \int_{a}^{b} \frac{g(x)}{x} d x-\int_{a}^{b} \frac{f(x) g(x)}{x} d x \\
& \quad=\frac{\ln b-\ln a}{2} \int_{0}^{1}\left(\int_{a}^{V(t)} \frac{g(x)}{x} d x\right)\left[V(t) f^{\prime}(V(t))-U(t) f^{\prime}(U(t))\right] d t .
\end{aligned}
$$

Proof. Let

$$
\begin{aligned}
& I_{1}:=\int_{0}^{1}\left(\int_{a}^{V(t)} \frac{g(x)}{x} d x\right) V(t) f^{\prime}(V(t)) d t, \\
& I_{2}:=\int_{0}^{1}\left(\int_{a}^{V(t)} \frac{g(x)}{x} d x\right) U(t) f^{\prime}(U(t)) d t .
\end{aligned}
$$

Since $g:[a, b] \rightarrow[0, \infty)$ is geometrically symmetric to $\sqrt{a b}$, we have $g(U(t))=$ $g(V(t))$ and

$$
\int_{a}^{V(t)} \frac{g(x)}{x} d x=\int_{U(t)}^{b} \frac{g(x)}{x} d x, \quad t \in[0,1] .
$$

Now we observe that

$$
\begin{aligned}
I_{1} & =\int_{0}^{1}\left(\int_{a}^{V(t)} \frac{g(x)}{x} d x\right) V(t) f^{\prime}(V(t)) d t \\
& =-\frac{2}{\ln b-\ln a} \int_{0}^{1}\left(\int_{a}^{V(t)} \frac{g(x)}{x} d x\right) d[f(V(t))] \\
& =-\left.\frac{2}{\ln b-\ln a}\left(\int_{a}^{V(t)} \frac{g(x)}{x} d x\right) f(V(t))\right|_{0} ^{1}-\int_{0}^{1} g(V(t)) f(V(t)) d t
\end{aligned}
$$




$$
\begin{aligned}
& =\frac{2 f(\sqrt{a b})}{\ln b-\ln a} \int_{a}^{\sqrt{a b}} \frac{g(x)}{x} d x-\int_{0}^{1} g(V(t)) f(V(t)) d t \\
& =\frac{2 f(\sqrt{a b})}{\ln b-\ln a} \int_{a}^{\sqrt{a b}} \frac{g(x)}{x} d x-\frac{2}{\ln b-\ln a} \int_{a}^{\sqrt{a b}} \frac{g(x) f(x)}{x} d x
\end{aligned}
$$

and, similarly,

$$
-I_{2}=\frac{2 f(\sqrt{a b})}{\ln b-\ln a} \int_{\sqrt{a b}}^{b} \frac{g(x)}{x} d x-\frac{2}{\ln b-\ln a} \int_{\sqrt{a b}}^{b} \frac{g(x) f(x)}{x} d x .
$$

Adding these equations and multiplying the result by $\frac{\ln b-\ln a}{2}$, we get the required identity.

Lemma 2. For $u, v>0$, we have

$$
\begin{gathered}
\zeta(u, v):=\int_{0}^{1} u^{(1-t) / 2} v^{(1+t) / 2} d t= \begin{cases}\sqrt{v} L(\sqrt{u}, \sqrt{v}), & u \neq v, \\
u, & u=v,\end{cases} \\
\xi(u, v):=\frac{1}{2} \int_{0}^{1} t u^{(1-t) / 2} v^{(1+t) / 2} d t= \begin{cases}\frac{v-\sqrt{v} L(\sqrt{u}, \sqrt{v})}{\ln v-\ln u}, & u \neq v \\
u / 4, & u=v,\end{cases}
\end{gathered}
$$

and

$$
\varsigma(u, v):=\frac{1}{2} \int_{0}^{1} t^{2} u^{(1-t) / 2} v^{(1+t) / 2} d t= \begin{cases}\frac{4 \sqrt{v} L(\sqrt{u}, \sqrt{v})-4 v+v(\ln v-\ln u)}{(\ln v-\ln u)^{2}}, & u \neq v, \\ u / 6, & u=v .\end{cases}
$$

Proof. The proof follows from a straightforward computation.

We now establish new Fejér type inequalities for GA-convex functions, which provide a weighted generalization of some results from [4].

Theorem 3. Let $f: I \rightarrow \mathbb{R}, I \subseteq \mathbb{R}_{+}$, be a differentiable function on $I^{\circ}$ and let $a, b \in I^{\circ}$ with $a<b$. Suppose that $g:[a, b] \rightarrow[0, \infty)$ is a continuous, positive, and geometrically symmetric to $\sqrt{a b}$ mapping. If $f^{\prime} \in L([a, b])$ and $\left|f^{\prime}\right|^{q}$ is GA-convex on $[a, b]$ for $q \geq 1$, then

$$
\begin{aligned}
\mid \mathcal{F} & (f, g ; a, b) \mid \leq \frac{(\ln b-\ln a)^{2}}{4}\|g\|_{\infty}\left\{[\zeta(a, b)-2 \xi(a, b)]^{1-1 / q}\right. \\
& \times\left([\zeta(a, b)+2 \varsigma(a, b)] A\left(\left|f^{\prime}(a)\right|^{q},\left|f^{\prime}(b)\right|^{q}\right)-2 \xi(a, b)\left|f^{\prime}(a)\right|^{q}\right)^{1 / q} \\
& +[\zeta(b, a)-2 \xi(b, a)]^{1-1 / q}([\zeta(b, a)+2 \varsigma(b, a)] \\
& \left.\left.\times A\left(\left|f^{\prime}(a)\right|^{q},\left|f^{\prime}(b)\right|^{q}\right)-2 \xi(b, a)\left|f^{\prime}(b)\right|^{q}\right)^{1 / q}\right\}
\end{aligned}
$$


where $\|g\|_{\infty}=\sup _{x \in[a, b]} g(x)<\infty$ and $\zeta(\cdot, \cdot), \xi(\cdot, \cdot), \varsigma(\cdot, \cdot)$ are defined in Lemma 2.

Proof. From Lemma 1, using Hölder's inequality, we have $|\mathcal{F}(f, g ; a, b)|$

$$
\begin{aligned}
\leq & \frac{\ln b-\ln a}{2} \int_{0}^{1}\left(\int_{a}^{V(t)} \frac{g(x)}{x} d x\right)\left[U(t)\left|f^{\prime}(U(t))\right|+V(t)\left|f^{\prime}(V(t))\right|\right] d t \\
\leq & \frac{(\ln b-\ln a)^{2}}{4}\|g\|_{\infty} \int_{0}^{1}\left[(1-t) U(t)\left|f^{\prime}(U(t))\right|+(1-t) V(t) f^{\prime}(V(t))\right] d t \\
\leq & \frac{(\ln b-\ln a)^{2}}{4}\|g\|_{\infty}\left\{\left(\int_{0}^{1}(1-t) U(t) d t\right)^{1-1 / q}\right. \\
& \times\left(\int_{0}^{1}(1-t) U(t)\left|f^{\prime}(U(t))\right|^{q} d t\right)^{1 / q} \\
& \left.+\left(\int_{0}^{1}(1-t) V(t) d t\right)^{1-1 / q}\left(\int_{0}^{1}(1-t) V(t)\left|f^{\prime}(V(t))\right|^{q} d t\right)^{1 / q}\right\} .
\end{aligned}
$$

By the GA-convexity of $\left|f^{\prime}\right|^{q}$ on $[a, b]$ for $q \geq 1$, and by Lemma 2, we have

$$
\begin{aligned}
& \int_{0}^{1}(1-t) U(t)\left|f^{\prime}(U(t))\right|^{q} \leq\left|f^{\prime}(a)\right|^{q} \int_{0}^{1}(1-t)\left(\frac{1-t}{2}\right) a^{(1-t) / 2} b^{(1+t) / 2} d t \\
& +\left|f^{\prime}(b)\right|^{q} \int_{0}^{1}(1-t)\left(\frac{1+t}{2}\right) a^{(1-t) / 2} b^{(1+t) / 2} d t \\
& =\left[\frac{1}{2} \zeta(a, b)+\varsigma(a, b)\right]\left|f^{\prime}(b)\right|^{q}+\left[\frac{1}{2} \zeta(a, b)-2 \xi(a, b)+\varsigma(a, b)\right]\left|f^{\prime}(a)\right|^{q}
\end{aligned}
$$

and, similarly,

$$
\begin{aligned}
& \int_{0}^{1}(1-t) V(t)\left|f^{\prime}(V(t))\right|^{q} \\
& \leq\left[\frac{1}{2} \zeta(b, a)+\varsigma(b, a)\right]\left|f^{\prime}(a)\right|^{q}+\left[\frac{1}{2} \zeta(b, a)-2 \xi(b, a)+\varsigma(b, a)\right]\left|f^{\prime}(b)\right|^{q} .
\end{aligned}
$$

Using (2.2) and (2.3) in (2.1), we get the required result.

Corollary 1. Suppose that the assumptions of Theorem 3 are satisfied and $q=1$. Then the following inequality holds:

$$
\begin{aligned}
& |\mathcal{F}(f, g ; a, b)| \\
& \leq 2\|g\|_{\infty}\left\{\left[L(a, b)-\sqrt{a} A(\sqrt{a}, \sqrt{b})-\frac{1}{4} a(\ln b-\ln a)\right]\left|f^{\prime}(a)\right|\right.
\end{aligned}
$$




$$
\left.+\left[L(a, b)-\sqrt{b} A(\sqrt{a}, \sqrt{b})+\frac{1}{4} b(\ln b-\ln a)\right]\left|f^{\prime}(b)\right|\right\} .
$$

Corollary 2. If $g(x)=\frac{1}{\ln b-\ln a}, x \in[a, b]$, in Theorem 3, then

$$
\begin{aligned}
\mid \mathcal{F} & (f, g ; a, b) \mid \leq \frac{(\ln b-\ln a)}{4}\left\{[\zeta(a, b)-2 \xi(a, b)]^{1-1 / q}\right. \\
& \times\left([\zeta(a, b)+2 \varsigma(a, b)] A\left(\left|f^{\prime}(a)\right|^{q},\left|f^{\prime}(b)\right|^{q}\right)-2 \xi(a, b)\left|f^{\prime}(a)\right|^{q}\right)^{1 / q} \\
& +[\zeta(b, a)-2 \xi(b, a)]^{1-1 / q}([\zeta(b, a)+2 \varsigma(b, a)] \\
& \left.\left.\times A\left(\left|f^{\prime}(a)\right|^{q},\left|f^{\prime}(b)\right|^{q}\right)-2 \xi(b, a)\left|f^{\prime}(b)\right|^{q}\right)^{1 / q}\right\} .
\end{aligned}
$$

Corollary 3. If $q=1$ in Corollary 2, then we get the inequality

$$
\begin{aligned}
& |\mathcal{F}(f, g ; a, b)| \leq \frac{2}{\ln b-\ln a} \\
& \quad \times\left\{\left[L(a, b)-\sqrt{a} A(\sqrt{a}, \sqrt{b})-\frac{1}{4} a(\ln b-\ln a)\right]\left|f^{\prime}(a)\right|\right. \\
& \left.\quad+\left[L(a, b)-\sqrt{b} A(\sqrt{a}, \sqrt{b})+\frac{1}{4} b(\ln b-\ln a)\right]\left|f^{\prime}(b)\right|\right\} .
\end{aligned}
$$

Theorem 4. Let $f$ and $g$ be the same as in Theorem 3. If $f^{\prime} \in L([a, b])$ and $\left|f^{\prime}\right|^{q}$ is GA-convex on $[a, b]$ for $q>1$, then

$$
\begin{aligned}
\mid \mathcal{F}(f, & , g ; a, b) \mid \leq \frac{(\ln b-\ln a)^{2-1 / q}\|g\|_{\infty}}{4 \cdot q^{1 / q}} \\
& \times\left(\frac{q-1}{2 q-1}\right)^{1-\frac{1}{q}}\left\{b ^ { 1 / 2 } \left(\left[L\left(a^{q / 2}, b^{q / 2}\right)-a^{q / 2}\right]\left|f^{\prime}(a)\right|^{q}\right.\right. \\
& \left.+\left[2 b^{q / 2}-a^{q / 2}-L\left(a^{q / 2}, b^{q / 2}\right)\right]\left|f^{\prime}(b)\right|^{q}\right)^{1 / q} \\
& +a^{1 / 2}\left(\left[L\left(a^{q / 2}, b^{q / 2}\right)+b^{q / 2}-2 a^{q / 2}\right]\left|f^{\prime}(a)\right|^{q}\right. \\
& \left.\left.+\left[b^{q / 2}-L\left(a^{q / 2}, b^{q / 2}\right)\right]\left|f^{\prime}(b)\right|^{q}\right)^{1 / q}\right\} .
\end{aligned}
$$

Proof. From Lemma 1, by Hölder's inequality we have

$$
\begin{aligned}
& |\mathcal{F}(f, g ; a, b)| \leq \frac{(\ln b-\ln a)^{2}\|g\|_{\infty}}{4}\left(\int_{0}^{1}(1-t)^{q /(q-1)} d t\right)^{1-1 / q} \\
& \times\left\{\left(\int_{0}^{1}[U(t)]^{q}\left|f^{\prime}(U(t))\right|^{q} d t\right)^{1 / q}+\left(\int_{0}^{1}[V(t)]^{q}\left|f^{\prime}(V(t))\right|^{q} d t\right)^{1 / q}\right\} .
\end{aligned}
$$


One has

$$
\begin{aligned}
\int_{0}^{1}[U(t)]^{q}\left|f^{\prime}(U(t))\right|^{q} \\
\leq\left|f^{\prime}(a)\right|^{q} \int_{0}^{1}\left(\frac{1-t}{2}\right) a^{[q(1-t)] / 2} b^{[q(1+t)] / 2} d t \\
\quad+\left|f^{\prime}(b)\right|^{q} \int_{0}^{1}\left(\frac{1+t}{2}\right) a^{[q(1-t)] / 2} b^{[q(1+t)] / 2} d t \\
=\frac{b^{q / 2}\left[L\left(a^{q / 2}, b^{q / 2}\right)-a^{q / 2}\right]}{q(\ln b-\ln a)}\left|f^{\prime}(a)\right|^{q} \\
\quad+\frac{b^{q / 2}\left[2 b^{q / 2}-a^{q / 2}-L\left(a^{q / 2}, b^{q / 2}\right)\right]}{q(\ln b-\ln a)}\left|f^{\prime}(b)\right|^{q}
\end{aligned}
$$

and, similarly,

$$
\begin{aligned}
\int_{0}^{1}[V(t)]^{q}\left|f^{\prime}(V(t))\right|^{q} \leq & \frac{a^{q / 2}\left[L\left(a^{q / 2}, b^{\frac{q}{2}}\right)+b^{q / 2}-2 a^{q / 2}\right]}{q(\ln b-\ln a)}\left|f^{\prime}(a)\right|^{q} \\
& +\frac{a^{q / 2}\left[b^{q / 2}-L\left(a^{q / 2}, b^{q / 2}\right)\right]}{q(\ln b-\ln a)}\left|f^{\prime}(b)\right|^{q}
\end{aligned}
$$

The inequality (2.4) is proved by applying (2.6) and (2.7) in (2.5).

Corollary 4. If the assumptions of Theorem 4 are satisfied and $g(x)=$ $\frac{1}{\ln b-\ln a}$ for all $x \in[a, b]$, then

$$
\begin{aligned}
|\mathcal{F}(f, g ; a, b)| & \\
\leq & \frac{(\ln b-\ln a)^{1-1 / q}}{4 \cdot q^{1 / q}}\left(\frac{q-1}{2 q-1}\right)^{1-\frac{1}{q}}\left\{b ^ { 1 / 2 } \left(\left[L\left(a^{q / 2}, b^{q / 2}\right)-a^{q / 2}\right]\left|f^{\prime}(a)\right|^{q}\right.\right. \\
& \left.+\left[2 b^{q / 2}-a^{q / 2}-L\left(a^{q / 2}, b^{q / 2}\right)\right]\left|f^{\prime}(b)\right|^{q}\right)^{1 / q}+a^{1 / 2}\left(\left[L\left(a^{q / 2}, b^{q / 2}\right)\right.\right. \\
& \left.\left.\left.+b^{q / 2}-2 a^{q / 2}\right]\left|f^{\prime}(a)\right|^{q}+\left[b^{q / 2}-L\left(a^{q / 2}, b^{q / 2}\right)\right]\left|f^{\prime}(b)\right|^{q}\right)^{1 / q}\right\} .
\end{aligned}
$$

Theorem 5. Let $f$ and $g$ be the same as in Theorem 3. If $f^{\prime} \in L([a, b])$ and $\left|f^{\prime}\right|^{q}$ is GA-convex on $[a, b]$ for $q>1$, then

$$
\begin{aligned}
& |\mathcal{F}(f, g ; a, b)| \leq \frac{(\ln b-\ln a)^{2-1 / q}}{2 \cdot(4 q)^{1 / q}}\|g\|_{\infty}\left(\frac{q-1}{2 q-1}\right)^{1-\frac{1}{q}} \\
& \quad \times\left\{\left[L\left(a^{q}, b^{q}\right)-a^{q}\right]\left|f^{\prime}(a)\right|^{q}+\left[b^{q}-L\left(a^{q}, b^{q}\right)\right]\left|f^{\prime}(b)\right|^{q}\right\}^{1 / q} .
\end{aligned}
$$

Proof. From Lemma 1 and Hölder's inequality it follows that (2.5) holds. By the power-mean inequality $a^{r}+b^{r} \leq 2^{1-r}(a+b)^{r}$ for $a>0, b>0$ and 
$r<1$, we have

$$
\begin{aligned}
& \left(\int_{0}^{1}[U(t)]^{q}\left|f^{\prime}(U(t))\right|^{q} d t\right)^{1 / q}+\left(\int_{0}^{1}[V(t)]^{q}\left|f^{\prime}(V(t))\right|^{q} d t\right)^{1 / q} \\
& \leq 2^{1-1 / q}\left(\int_{0}^{1}[U(t)]^{q}\left|f^{\prime}(U(t))\right|^{q} d t+\int_{0}^{1}[V(t)]^{q}\left|f^{\prime}(V(t))\right|^{q} d t\right)^{1 / q}
\end{aligned}
$$

Since $\left|f^{\prime}\right|^{q}$ is GA-convex on $[a, b]$ for $q>1$, by (2.6) and (2.7) we get

$$
\begin{aligned}
& \int_{0}^{1}[U(t)]^{q}\left|f^{\prime}(U(t))\right|^{q} d t+\int_{0}^{1}[V(t)]^{q}\left|f^{\prime}(L(t))\right|^{q} d t \\
& \quad \leq\left[\frac{2 L\left(a^{q}, b^{q}\right)-2 a^{q}}{q(\ln b-\ln a)}\right]\left|f^{\prime}(a)\right|^{q}+\left[\frac{2 b^{q}-2 L\left(a^{q}, b^{q}\right)}{q(\ln b-\ln a)}\right]\left|f^{\prime}(b)\right|^{q} .
\end{aligned}
$$

Applying (2.9) and (2.10) in (2.5), we obtain the required inequality (2.8).

Corollary 5. If the assumptions of Theorem 5 are satisfied and $g(x)=$ $\frac{1}{\ln b-\ln a}$ for all $x \in[a, b]$, then the following inequality holds:

$$
\begin{aligned}
& |\mathcal{F}(f, g ; a, b)| \leq \frac{(\ln b-\ln a)^{1-1 / q}}{2 \cdot(4 q)^{1 / q}}\|g\|_{\infty}\left(\frac{q-1}{2 q-1}\right)^{1-\frac{1}{q}} \\
& \quad \times\left\{\left[L\left(a^{q}, b^{q}\right)-a^{q}\right]\left|f^{\prime}(a)\right|^{q}+\left[b^{q}-L\left(a^{q}, b^{q}\right)\right]\left|f^{\prime}(b)\right|^{q}\right\}^{1 / q} .
\end{aligned}
$$

Theorem 6. By the assumptions of Theorem 5 we have the inequality

$$
\begin{aligned}
& |\mathcal{F}(f, g ; a, b)| \leq \frac{(\ln b-\ln a)^{2}\left[L\left(a^{q /[2(q-1)]}, b^{q /[2(q-1)]}\right)\right]^{1-1 / q}\|g\|_{\infty}}{8} \\
& \times\left\{\left(b^{1 / 2}\left(\frac{1}{2 q+1}\right)^{1 / q}+a^{1 / 2}\left[{ }_{2} F_{1}(-q, 1 ; q+2 ;-1) \cdot \frac{1}{q+1}\right]^{1 / q}\right)\left|f^{\prime}(a)\right|\right. \\
& \left.+\left(a^{1 / 2}\left(\frac{1}{2 q+1}\right)^{1 / q}+b^{1 / 2}\left[{ }_{2} F_{1}(-q, 1 ; q+2 ;-1) \cdot \frac{1}{q+1}\right]^{1 / q}\right)\left|f^{\prime}(b)\right|\right\} .
\end{aligned}
$$


Proof. As in the proof of Theorem 3, from Lemma 1 and the GA-convexity of $\left|f^{\prime}\right|$ on $[a, b]$ we have

$$
\begin{aligned}
& |\mathcal{F}(f, g ; a, b)| \leq \frac{(\ln b-\ln a)^{2}}{4}\|g\|_{\infty}\left\{\int _ { 0 } ^ { 1 } a ^ { ( 1 - t ) / 2 } b ^ { ( 1 + t ) / 2 } \left[(1-t)\left(\frac{1-t}{2}\right)\left|f^{\prime}(a)\right|\right.\right. \\
& \left.+(1-t)\left(\frac{1+t}{2}\right)\left|f^{\prime}(b)\right|\right] d t+\int_{0}^{1} a^{(1+t) / 2} b^{(1-t) / 2} \\
& \left.\times\left[(1-t)\left(\frac{1+t}{2}\right)\left|f^{\prime}(a)\right|+(1-t)\left(\frac{1-t}{2}\right)\left|f^{\prime}(b)\right|\right] d t\right\} .
\end{aligned}
$$

Using Hölder's integral inequality, we get

$$
\begin{aligned}
& \int_{0}^{1} a^{(1-t) / 2} b^{(1+t) / 2}\left[(1-t)\left(\frac{1-t}{2}\right)\left|f^{\prime}(a)\right|+(1-t)\left(\frac{1+t}{2}\right)\left|f^{\prime}(b)\right|\right] d t \\
& \leq\left(\int_{0}^{1} a^{[q(1-t)] /[2(q-1)]} b^{[q(1+t)] /[2(q-1)]} d t\right)^{1-1 / q} \\
& \times\left\{\left[\int_{0}^{1}(1-t)^{q}\left(\frac{1-t}{2}\right)^{q} d t\right]^{1 / q}\left|f^{\prime}(a)\right|+\left[\int_{0}^{1}(1-t)^{q}\left(\frac{1+t}{2}\right)^{q} d t\right]^{1 / q}\left|f^{\prime}(b)\right|\right\} \\
& =\frac{1}{2}\left[b^{q /[2(q-1)]} L\left(a^{q /[2(q-1)]}, b^{q /[2(q-1)]}\right)\right]^{1-1 / q} \\
& \times\left\{\left(\frac{1}{2 q+1}\right)^{1 / q}\left|f^{\prime}(a)\right|+\left[{ }_{2} F_{1}(-q, 1 ; q+2 ;-1) \cdot \frac{1}{q+1}\right]^{1 / q}\left|f^{\prime}(b)\right|\right\} .
\end{aligned}
$$

Similarly, we have

$$
\begin{aligned}
& \int_{0}^{1} a^{(1+t) / 2} b^{(1-t) / 2}\left[t\left(\frac{1+t}{2}\right)\left|f^{\prime}(a)\right|+t\left(\frac{1-t}{2}\right)\left|f^{\prime}(b)\right|\right] d t \\
& \leq \frac{1}{2}\left[a^{q /[2(q-1)]} L\left(a^{q /[2(q-1)]}, b^{q /[2(q-1)]}\right)\right]^{1-1 / q}\left\{\left(\frac{1}{2 q+1}\right)^{1 / q}\left|f^{\prime}(b)\right|\right. \\
& \left.+\left[{ }_{2} F_{1}(-q, 1 ; q+2 ;-1) \cdot \frac{1}{q+1}\right]^{1 / q}\left|f^{\prime}(a)\right|\right\} .
\end{aligned}
$$

Using (2.12) and (2.13) in (2.11), we obtain the required inequality.

Corollary 6. If $g(x)=\frac{1}{\ln b-\ln a}$ in Theorem 6 , then

$$
|\mathcal{F}(f, g ; a, b)| \leq \frac{(\ln b-\ln a)\left[L\left(a^{q /[2(q-1)]}, b^{q /[2(q-1)]}\right)\right]^{1-1 / q}}{2}
$$




$$
\begin{aligned}
& \times\left\{\left(b^{1 / 2}\left(\frac{1}{2 q+1}\right)^{1 / q}+a^{1 / 2}\left[{ }_{2} F_{1}(-q, 1 ; q+2 ;-1) \cdot \frac{1}{q+1}\right]^{1 / q}\right)\left|f^{\prime}(a)\right|\right. \\
& \left.+\left(a^{1 / 2}\left(\frac{1}{2 q+1}\right)^{1 / q}+b^{1 / 2}\left[{ }_{2} F_{1}(-q, 1 ; q+2 ;-1) \cdot \frac{1}{q+1}\right]^{1 / q}\right)\left|f^{\prime}(b)\right|\right\} .
\end{aligned}
$$

\section{Applications to special means}

In this section we apply some of the above established inequalities of Hermite-Hadamard type involving the product of a geometrically-arithmetically convex function and a geometrically symmetric function to construct inequalities for special means.

Let $f(x)=x^{r}$ for $x>0, r \in \mathbb{R}$ with $r \neq 0$. Then

$$
\begin{aligned}
\left|f^{\prime}\left(x^{\lambda} y^{1-\lambda}\right)\right|^{q} & =|r|^{q}\left[x^{q(r-1)}\right]^{\lambda}\left[y^{q(r-1)}\right]^{1-\lambda} \\
& \leq|r|^{q}\left[\lambda x^{q(r-1)}+(1-\lambda) y^{q(r-1)}\right]
\end{aligned}
$$

for $\lambda \in[0,1], x, y>0$ and $q \geq 1$. That is, $\left|f^{\prime}(x)\right|^{q}=|r|^{q} x^{q(r-1)}$ is geometrically-arithmetically convex on $[a, b]$ for $q \geq 1$ and $r \neq 1$, where $a, b>0$.

Let the function $g:[a, b] \rightarrow \mathbb{R}_{0}$ be defined by

$$
g(x)=\left(\frac{x}{\sqrt{a b}}-\frac{\sqrt{a b}}{x}\right)^{2}, x \in[a, b] .
$$

It is obvious that

$$
g\left(\frac{a b}{x}\right)=g(x)
$$

for all $x \in[a, b]$. Hence $g(x)$ is geometrically symmetric with respect to $x=\sqrt{a b}$.

Applications of our results are given in the following theorems.

Theorem 7. Let $0<a<b, r \in \mathbb{R} \backslash\{-2,0,1,2\}$, and $q \geq 1$. Then

$$
\begin{gathered}
\mathcal{G}[r ; a, b]:=\mid 2 G^{r-2}(a, b) L\left(a^{2}, b^{2}\right)+2 L\left(a^{r}, b^{r}\right)-2 G^{r}(a, b) \\
-G^{2}(a, b) L\left(a^{r-2}, b^{r-2}\right)+\frac{L\left(a^{r+2}, b^{r+2}\right)}{G^{2}(a, b)} \mid \\
\leq \frac{(b-a)|r|}{2 G(a, b)}\left\{[\sqrt{b} L(\sqrt{a}, \sqrt{b})-G(a, b)]^{1-1 / q}\right. \\
\times\left(\left[\frac{4 \sqrt{b} L(\sqrt{a}, \sqrt{b})-4 b}{\ln b-\ln a}-G(a, b)+2 b\right] A\left(a^{q(r-1)}, b^{q(r-1)}\right)\right.
\end{gathered}
$$




$$
\begin{gathered}
\left.-[b-\sqrt{b} L(\sqrt{a}, \sqrt{b})] a^{q(r-1)}\right)^{1 / q}+[G(a, b)-\sqrt{a} L(\sqrt{a}, \sqrt{b})]^{1-1 / q} \\
\times\left(\left[\frac{4 \sqrt{a} L(\sqrt{a}, \sqrt{b})-4 a}{\ln b-\ln a}+G(a, b)-2 a\right] A\left(a^{q(r-1)}, b^{q(r-1)}\right)\right. \\
\left.\left.+[a-\sqrt{a} L(\sqrt{a}, \sqrt{b})] b^{q(r-1)}\right)^{1 / q}\right\} .
\end{gathered}
$$

Proof. By applying Theorem 3 to the functions $f(x)=x^{r}, x>0$, and (3.1) we get the desired result.

Corollary 7. Suppose the assumptions of Theorem 7 are satisfied. If $r=-1$, then the following inequality holds:

$$
\begin{aligned}
& \mathcal{H}[a, b]:=\mid \frac{2 L\left(a^{2}, b^{2}\right)-2 G^{2}(a, b)+L(a, b) G(a, b)}{G^{3}(a, b)} \\
& -G^{2}(a, b) L\left(a^{-3}, b^{-3}\right)+2 L\left(a^{-1}, b^{-1}\right) \mid \\
& \leq \frac{(b-a)}{2 G(a, b)}\left\{[\sqrt{b} L(\sqrt{a}, \sqrt{b})-G(a, b)]^{1-1 / q}\right. \\
& \times\left(\left[\frac{4 \sqrt{b} L(\sqrt{a}, \sqrt{b})-4 b}{\ln b-\ln a}-G(a, b)+2 b\right] A\left(a^{-2 q}, b^{-2 q}\right)\right. \\
& \left.\left.-[b-\sqrt{b} L(\sqrt{a}, \sqrt{b})] a^{-2 q}\right)^{1 / q}+[G(a, b)-\sqrt{a} L(\sqrt{a}, \sqrt{b})]\right]^{1-1 / q} \\
& \times\left(\left[\frac{4 \sqrt{a} L(\sqrt{a}, \sqrt{b})-4 a}{\ln b-\ln a}+G(a, b)-2 a\right] A\left(a^{-2 q}, b^{-2 q}\right)\right. \\
& \left.\left.\quad+[a-\sqrt{a} L(\sqrt{a}, \sqrt{b})] b^{-2 q}\right)^{1 / q}\right\} .
\end{aligned}
$$

Corollary 8. Under the assumptions of Theorem \%, the following inequality holds true for $q=1$ :

$$
\begin{aligned}
\mathcal{G}[r ; a, b] \leq & \frac{(b-a)|r|}{2 G(a, b)}\left[\frac{8 A(\sqrt{a}, \sqrt{b}) L(\sqrt{a}, \sqrt{b})-8 A(a, b)}{\ln b-\ln a}\right. \\
& +2(b-a)+(r-2)(b-a) G^{2}(a, b) L_{r-3}^{r-3}(a, b) \\
& \left.-\left(r-\frac{3}{2}\right)(b-a) G(a, b) L_{r-\frac{5}{2}}^{r-\frac{5}{2}}(a, b)\right] .
\end{aligned}
$$


Corollary 9. If $r=-1$ in Corollary 8 , then we have the inequality

$$
\begin{aligned}
\mathcal{H}[a, b] \leq & \frac{(b-a)}{2}\left[\frac{8 A(\sqrt{a}, \sqrt{b}) L(\sqrt{a}, \sqrt{b})-8 A(a, b)}{(\ln b-\ln a) G(a, b)}+\frac{2(b-a)}{G(a, b)}\right. \\
& \left.-3(b-a) G(a, b) L_{-4}^{-4}(a, b)+\frac{5}{2}(b-a) L_{\frac{7}{2}}^{\frac{7}{2}}(a, b)\right] .
\end{aligned}
$$

Theorem 8. Let $0<a<b, r \in \mathbb{R} \backslash\{-2,0,1,2\}$, and $q>1$. Then

$$
\begin{aligned}
\mathcal{G}[r ; a, b] \leq & \frac{(\ln b-\ln a)^{1-1 / q}(b-a)|r|}{4 \cdot q^{1 / q} G(a, b)}\left(\frac{q-1}{2 q-1}\right)^{1-\frac{1}{q}} \\
\times & \left\{b ^ { 1 / 2 } \left(\left[L\left(a^{q / 2}, b^{q / 2}\right)-a^{q / 2}\right] a^{q(r-1)}\right.\right. \\
& \left.+\left[2 b^{q / 2}-a^{q / 2}-L\left(a^{q / 2}, b^{q / 2}\right)\right] b^{q(r-1)}\right)^{1 / q} \\
& +a^{1 / 2}\left(\left[L\left(a^{q / 2}, b^{q / 2}\right)+b^{q / 2}-2 a^{q / 2}\right] a^{q(r-1)}\right. \\
& \left.\left.+\left[b^{q / 2}-L\left(a^{q / 2}, b^{q / 2}\right)\right] b^{q(r-1)}\right)^{1 / q}\right\} .
\end{aligned}
$$

Proof. By applying Theorem 4 to the functions $f(x)=x^{r}$ and (3.1) we get the desired result.

Corollary 10. Suppose the assumptions of Theorem 8 are fulfilled. If $r=-1$, then the following inequality holds:

$$
\begin{aligned}
& \mathcal{H}[a, b] \leq \frac{(\ln b-\ln a)^{1-1 / q}(b-a)}{4 \cdot q^{1 / q} G(a, b)}\left(\frac{q-1}{2 q-1}\right)^{1-\frac{1}{q}} \\
& \times\left\{b^{1 / 2}\left(\left[L\left(a^{q / 2}, b^{q / 2}\right)-a^{q / 2}\right] a^{-2 q}+\left[2 b^{q / 2}-a^{q / 2}-L\left(a^{q / 2}, b^{q / 2}\right)\right] b^{-2 q}\right)^{1 / q}\right. \\
& \left.+a^{1 / 2}\left(\left[L\left(a^{q / 2}, b^{q / 2}\right)+b^{q / 2}-2 a^{q / 2}\right] a^{-2 q}+\left[b^{q / 2}-L\left(a^{q / 2}, b^{q / 2}\right)\right] b^{-2 q}\right)^{1 / q}\right\} .
\end{aligned}
$$

Theorem 9. Let $0<a<b, r \in \mathbb{R} \backslash\{-2,0,1,2\}$, and $q>1$. Then

$$
\begin{aligned}
\mathcal{G}[r ; a, b] \leq & \frac{(b-a)^{2}|r|}{2^{2 / q+1} L(a, b) G(a, b)}\left(\frac{q-1}{2 q-1}\right)^{1-\frac{1}{q}} \\
& \times\left\{r L\left(a^{q r}, b^{q r}\right)-(r-1) L\left(a^{q(r-1)}, b^{q(r-1)}\right) L\left(a^{q}, b^{q}\right)\right\}^{1 / q} .
\end{aligned}
$$

Proof. Application of Theorem 5 to the functions $f(x)=x^{r}$ and (3.1) gives the desired result. 
Corollary 11. By the assumptions of Theorem 9, for $r=-1$ we have the inequality

$$
\begin{aligned}
\mathcal{H}[a, b] \leq & \frac{(b-a)^{2}}{2^{2 / q+1} G(a, b) L(a, b)}\left(\frac{2 q-1}{q-1}\right)^{1-\frac{1}{q}} \\
& \times\left[2 L\left(a^{-2 q}, b^{-2 q}\right) L\left(a^{-1}, b^{-1}\right)-L\left(a^{-q}, b^{-q}\right)\right]^{1 / q} .
\end{aligned}
$$

Acknowledgments. The authors are very thankful for the anonymous referee for his/her very useful comments that have been incorporated in the final version of the manuscript.

\section{References}

[1] S. S. Dragomir, Inequalities of Hermite-Hadamard type for GA-convex functions, RGMIA Research Report Collection 18 (2015), Article 30, 20 pp.

[2] S. S. Dragomir, Some new inequalities of Hermite-Hadamard type for GA-convex functions, RGMIA Research Report Collection 18 (2015), Article 33, 14 pp.

[3] J. Hua, B.-Y. Xi, and F. Qi, Hemite-Hadamard type inequalities for geometricallyarithmetically s-convex functions, Commun. Korean Math. Soc. 29(1) (2014), 51-63.

[4] İ. İscan, Hemite-Hadamard type inequalities for $G A$-s-convex functions, Matematiche 69(2) (2014), 129-146.

[5] M. A. Latif, New Hermite-Hadamard type integral inequalities for GA-convex functions with applications, Analysis 34(4) (2014), 379-389.

[6] M. A. Latif, S. S. Dragomir, and E. Momoniat, Some Fejé r type integral inequalities for geometrically-arithmetically-convex functions with applications. (accepted)

[7] C. P. Niculescu, Convexity according to the geometric mean, Math. Inequal. Appl. 3(2) (2000), 155-167.

[8] C. P. Niculescu, Convexity according to means, Math. Inequal. Appl. 6(4) (2003), 571-579.

[9] Y. Shuang, H. P. Yin, and F. Qi, Hermite-Hadamard type integral inequalities for geometric-arithmetically s-convex functions, Analysis 33, (2013), 1001-1010.

[10] T.-Y. Zhang, A.-P. Ji, and F. Qi, Some inequalities of Hermite-Hadamard type for GA-Convex functions with applications to means, Matematiche 48(1) (2013), 229-239.

School of Engineering and Science, Victoria University, PO Box 14428 Melbourne City, MC 8001, Australia; School of Computational and Applied Mathematics, University of the Witwatersrand, Private Bag 3, Wits 2050, JohanNESBURG, SOUTh AFricA

E-mail address: sever.dragomir@vu.edu.au

Department of Basic Sciences, Deanship of Preparatory Year, University of Hail, Hail 2440, Saudi Arabia

E-mail address: m_amer_latif@hotmail.com

Department of Pure and Applied Mathematics, University of Johannesburg, P.O. Box 524, Auckland Park 2006, South Africa

E-mail address: ebrahim.momoniat@gmail.com 